\title{
Respiratory antibacterial prescribing in primary care and the COVID-19 pandemic in England, winter season 2020-21
}

\author{
Amelia Andrews $\mathbb{D}^{1,2,3 *}$, Sabine Bou-Antoun ${ }^{1,4}$, Rebecca Guy ${ }^{1}$, Colin S. Brown ${ }^{1,4}$, Susan Hopkins $\mathbb{D}^{1,2,4}$ and \\ Sarah Gerver ${ }^{1,2,4}$ \\ ${ }^{1}$ HCAI, Fungal, AMR, AMU \& Sepsis Division, UK Health Security Agency, London NW9 5EQ, UK; ${ }^{2}$ National Institute for Health Research \\ (NIHR) Health Protection Research Unit in Healthcare Associated Infections and Antimicrobial Resistance at the University of Oxford, \\ Oxford OX3 9DU, UK; ${ }^{3}$ Nuffield Department of Medicine, University of Oxford, Oxford OX3 9DU, UK; ${ }^{4}$ National Institute for Health \\ Research (NIHR) Health Protection Research Unit in Healthcare Associated Infections and Antimicrobial Resistance at Imperial College \\ London, London W12 ONN, UK \\ *Corresponding author. E-mail: Amelia.Andrews@phe.gov.uk
}

Received 2 September 2021; accepted 12 November 2021

\begin{abstract}
Background: Antibacterial prescribing for respiratory tract infections (RTIs) accounts for almost half of all prescribing in primary care. Nearly a quarter of antibacterial prescribing in primary care is estimated to be inappropriate, the greatest being for RTIs. The COVID-19 pandemic has changed the provision of healthcare services and impacted the levels of antibacterials prescribed.
\end{abstract}

Objectives: To describe the changes in community antibacterial prescribing for RTIs in winter 2020-21 in England.

Methods: RTI antibacterial prescribing was measured in prescription items/1000 population for primary care from January 2014 and in DDDs/1000 population/day for the totality of RTI prescribing [combined with Accident \& Emergency (A\&E) in secondary care], from January 2016 to February 2021. Trends were assessed using negative binomial regression and seasonally adjusted interrupted time-series analysis.

Results: Antibacterials prescribed for RTIs reduced by a further $12.4 \%$ per season compared with pre-COVID $(P<0.001)$. In winter 2020-21, RTI prescriptions almost halved compared with the previous winter in 2019-20 $(P<0.001)$. The trend observed for total RTI prescribing (primary care with A\&E) was similar to that observed in the community alone.

Conclusions: During COVID-19, RTI prescribing reduced in the community and the expected rise in winter was not seen in 2020-21. We found no evidence that RTI prescribing shifted from primary care to A\&E in secondary care. The most likely explanation is a decrease in RTIs and presentations to primary care associated with national prevention measures for COVID-19.

\section{Introduction}

Antibacterial prescribing in primary care accounts for $70 \%$ of total prescribing in England, ${ }^{1}$ with $46 \%$ prescribed for respiratory tract infections (RTIs). ${ }^{2}$ Prudent antimicrobial prescribing has been encouraged by the National Health Service (NHS) quality improvement schemes because inappropriate antimicrobial use is directly linked to antimicrobial resistance (AMR), which is a global public health issue. ${ }^{3,4}$ Following effective antimicrobial stewardship interventions, prescribing in primary care has fallen by $14 \%$ between 2015 and 2019. ${ }^{1}$

Approximately $23 \%$ of antibacterial prescribing in primary care was identified as inappropriate, with the greatest area for improvement relating to RTIs. ${ }^{5}$ RTI indications are largely selflimiting, often caused by viral pathogens and adverse complications are rare. ${ }^{6,7}$ Antibacterials are frequently prescribed regardless of this, partly due to patient expectations and difficulties in clinically distinguishing between viral and bacterial RTIs without rapid diagnostics in the community.

The SARS-CoV-2 coronavirus (COVID-19) pandemic has challenged antimicrobial stewardship programmes with similar clinical features being seen in both SARS-CoV-2 severe respiratory infections and bacterial pneumonia. Thus, levels of antibacterial prescribing among hospitalized COVID-19 patients were elevated, ${ }^{10}$ although studies have shown that bacterial co-infections in COVID-19 patients were low. ${ }^{11,12}$ In England, overall community 
antibacterial prescribing decreased but prescribing for communityacquired pneumonia increased at the beginning of COVID-19. ${ }^{13}$ COVID-19 has also changed the delivery of healthcare services, with a fall in General Practice (GP) attendance in March 2020.14 With limited community diagnostics, the UK government developed a direct-to-citizen national laboratory with the capacity for more than 500000 SARS-CoV-2 tests-per-day. ${ }^{15}$ Here we aim describe the changes in prescribing for the treatment of RTIs in the community in 2020-21.

\section{Methods}

\section{Data sources}

National-level antibacterial prescribing data were extracted from the NHS Business Services Authority (via ePACT2) and IQVIA (database for drugs dispensed from NHS hospital pharmacy systems) for primary and secondary care, respectively. Primary care prescribing included all antibacterials (within British National Formulary chapter 5.1), except anti-tuberculosis and anti-leprotic drugs, for all Clinical Commissioning Groups in England from dispensed NHS prescriptions in the community, including GPs, out-of-hours and urgent care. Secondary care prescribing included all antibacterials for systemic use (from the World Health Organization's DDD index) prescribed within the Accident \& Emergency (A\&E) speciality for all patients cared for in English NHS acute hospitals. Monthly data were extracted from January 2014 for primary care (5 year age-group data from April 2015) and from January 2016 for secondary care, to February 2021.

Population estimates were obtained from the Office for National Statistics; 2020 was used as a proxy for 2021.

\section{Definitions and data analysis}

Antibacterials recommended for treatment of self-limiting RTIs (cough, otitis media, sinusitis and sore throat) were defined based on the National Institute for Health and Care Excellence guidelines ${ }^{7}$ (see Table S1, available as Supplementary data at JAC Online).

The COVID-19 pandemic period was defined as spring 2020-21 onwards. Seasons were defined as spring (March-May), summer (JuneAugust), autumn (September-November) and winter (DecemberFebruary).

Antibacterial consumption was measured as prescription items/1000 population in primary care. Trends were assessed using negative binomial regression, season was adjusted for as an independent variable. Seasonally adjusted interrupted time-series analysis was used to evaluate changes in RTI prescribing in the community after the pandemic (the 'intervention'), by using the underlying trend before COVID-19 (the counterfactual) to predict the 2020-21 trend in the absence of COVID-19. Statistical significance was attributed when $P<0.05$.

A joint assessment of prescribing in primary care and $A \& E$ (also known as the Emergency Department) in secondary care was performed in the descriptive analysis. Although prescription item is the preferred metric in primary care, it is not available for secondary care. Thus, the joint prescribing data were translated into DDDs and expressed as DDDs/1000 population/ day as a measure of antibiotic use in combined settings, from January 2016 to February 2021.

Stata 15 was used for all analyses (StataCorp, TX, USA).

\section{Results}

\section{Primary care}

The recommended antibacterials for the treatment of RTI account for over half of all antibacterial items prescribed in the community

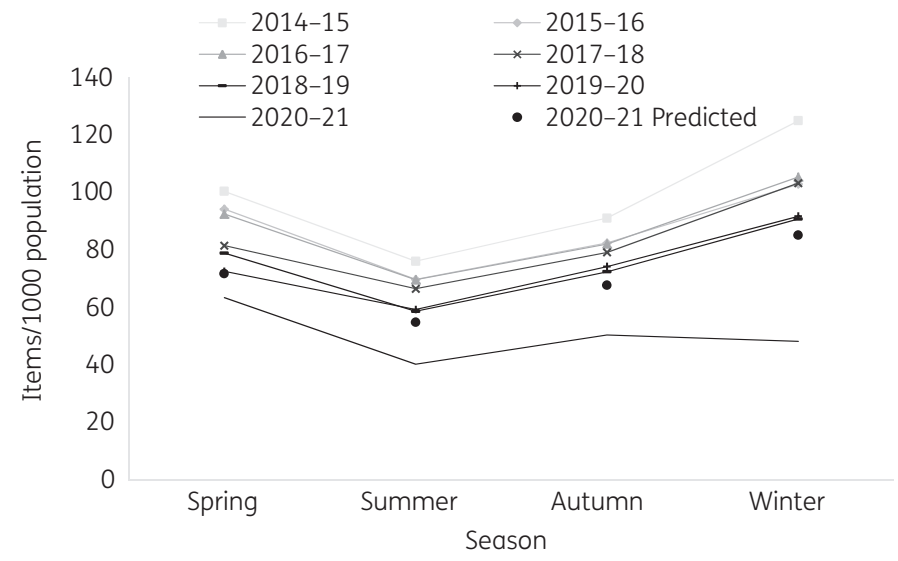

Figure 1. Interrupted time-series for all RTI antibacterial prescription items prescribed/1000 population in primary care by season, showing the counterfactual scenario (in black dots), England, 2014-15 to 202021.

annually (Table S2). RTI antibacterials reduced by a further $12.4 \%$ per season compared with pre-COVID (95\% CI: $-15.7 \%$ to $-8.9 \%$, $P<0.001$ ), with the largest annual drop seen between 2019-20 and 2020-21 (Figure 1). Before 2020-21, an uptick in antibacterial items recommended for RTI treatment was observed every winter, compared with other seasons $(P<0.001)$; however, this was not observed in winter 2020-21 (Figure 1). RTI prescriptions almost halved compared with the previous winter ( 48.4 in 2020-21 versus 92.0 items per 1000 population in 2019-20, $P<0.001$ ) (Table S2).

The previous occurrence of seasonal winter peaks prior to 2020-21 was observed in all age-groups $(P<0.01)$, as was the absence of the winter peak in recommended RTI antibacterials prescriptions in 2020-21 ( $P<0.001)$; a $>70 \%$ reduction between winter 2019-20 and winter 2020-21 was present in infants and children (under 14 years old) (Figure S1).

For the recommended RTI antibacterials, oral amoxicillin and doxycycline prescriptions were predominant in the observed seasonal rises in the winters before 2020-21. In winter 2020-21, oral amoxicillin prescriptions saw the largest decrease (by $60.1 \%$ ) compared with winter 2019-20 (Figure S2).

\section{Total RTI prescribing}

Antibacterial prescribing recommended for RTIs in primary care was combined with that of hospital A\&E to account for possible shifts in health-seeking behaviours and to non-guidance RTI antibacterials. The observed total trend was similar to the trend for community prescribing alone. This includes the large drop in prescribing for antibacterials recommended for RTI treatment in February 2021 (by -36.6\%), compared with the same month in the previous year (Figure 2 and Figure $S 3$ for $A \& E$ only). No change was observed in winter 2020/21 for all other antibacterials that were not recommended for RTI treatment (Table S3).

\section{Discussion}

In England, antibacterials recommended for RTI treatment prescribed in the community reduced during the COVID-19 pandemic in 2020-21. Before COVID-19, winter typically saw the greatest 


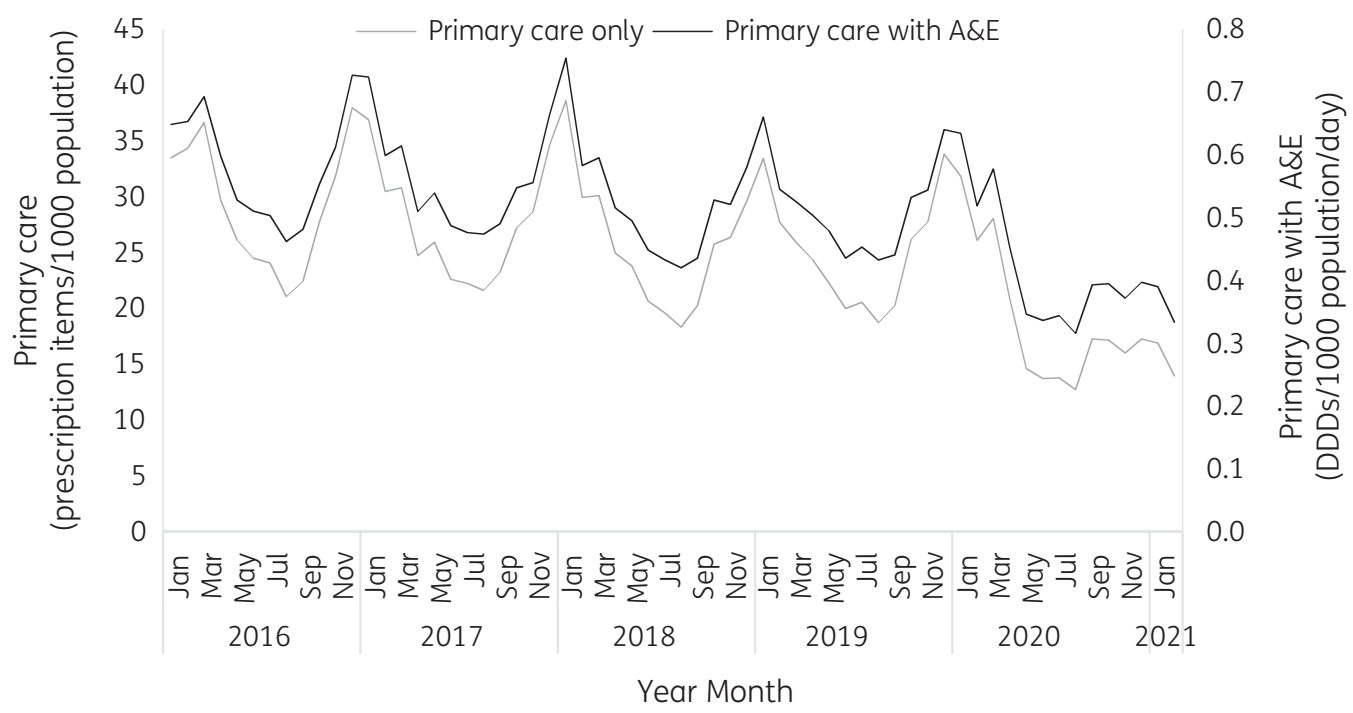

Figure 2. All RTI antibacterials prescribed in primary care (prescription items/1000 population) and primary care with A\&E (DDDs/1000 population/ day), England, January 2016 to February 2021.

prescribing of RTI antibacterials annually, coinciding with the seasonal rise in respiratory infections circulating. ${ }^{16}$ However, the expected rise in prescribing for RTIs in primary care was not observed in winter 2020-21.

The pandemic has undoubtedly affected the provision of healthcare services, with overall GP appointments falling in early $2020 .{ }^{14}$ Patients may have sought remote health advice, whilst some may have avoided attending healthcare centres entirely owing to concerns regarding COVID-19. It is also possible that those with RTI symptoms may have attended A\&E and bypassed primary care, potentially due to the more severe symptoms presented at the point of seeking healthcare, having avoided healthcare settings earlier. We have shown, however, after accounting for possible changes in healthcare service provision, the totality of RTI prescribing did not increase to previous baseline levels during COVID-19. This is supported by the lower than expected rates of $A \& E$ attendance for patients with acute respiratory infection seen in syndromic surveillance. ${ }^{17}$

The COVID-19 pandemic saw drastic reductions in person-toperson contact that were associated with national infection prevention measures ('lockdown' periods and social distancing), which may have reduced the transmission of infectious pathogens besides SARS-CoV-2. Syndromic surveillance showed the incidence of upper and lower RTIs in GP in-hours services was substantially lower than the levels that would have been expected prior to COVID-19, especially during the winter months. ${ }^{18}$ Furthermore, greatly reduced consultation rates for RTI and urinary tract infections were recorded early on in the pandemic, especially in younger age-groups. ${ }^{14}$

RTI antibacterial prescribing saw a large decrease for infants and young children. Given that the prescribing of oral amoxicillin in young children is usually high in the winter ${ }^{13}$ and inappropriate prescribing is often associated with this age-group, overall amoxicillin prescriptions also decreased in the community. Although early years settings were kept open during winter 2020-21, COVID-19 restrictions are likely to have resulted in less social interactions between children compared with usual activity, and thus may have limited the transmission of communicable respiratory infections.

With large reductions in RTI antibacterial prescribing seen in primary care during COVID-19 and some evidence of small reductions in the proportion of RTI consultations with antibiotics prescribed, ${ }^{14}$ there is an opportunity to evaluate prescribing supported by diagnostic tests for respiratory infections. Rapid diagnostics in the community, such as lateral flow tests, have been effective in detecting SARS-CoV-2 and are quicker and cheaper than PCR tests. ${ }^{19}$ Therefore, the development of and access to quick and reliable point-of-care diagnostics are key tools to reduce AMR and are part of the ambitions set out in the UK National Action Plan. ${ }^{3}$ Diagnostics to differentiate viral and bacterial aetiologies ${ }^{20}$ could spare unnecessary antibacterial use and reduce GP attendance in future winters.

Limitations of this study are its ecological nature; we were unable to identify indications for the antibacterials prescribed. Patient-level prescribing with consultation data would be useful to assess the trends observed. This would allow linkage to antimicrobial susceptibility results and thus investigations into the change in RTI antibacterial use and the impact on AMR.

\section{Conclusions}

Antibacterial prescribing for treatment of RTIs in the community reduced in 2020-21, during the COVID-19 pandemic. The expected rise in community prescribing for RTIs in winter was not observed in winter 2020-21 and prescribing did not appear to have shifted to $A \& E$ in secondary care. The most likely explanation is a decrease in RTIs and presentations to primary care associated with COVID19 prevention measures.

\section{Acknowledgements}

National antibiotic consumption data for secondary care: data are provisional for up to 2 years and copyright IQVIA Solutions UK Limited and its 
affiliates (all rights reserved). Use for sales, marketing or any other commercial purposes is not permitted without IQVIA Solutions UK Limited's express prior written consent.

We would like to thank Ross Harris for providing statistical advice.

\section{Funding}

This work was carried out as enhanced surveillance of the routine work of the UK Health Security Agency (UKHSA), formerly Public Health England.

A. A. is funded by the National Institute for Health Research (NIHR) Health Protection Research Unit (HPRU) in Healthcare Associated Infections and Antimicrobial Resistance, a partnership between the UKHSA and the University of Oxford [NIHR200915]. S. H., C. B. and S. G. are supported by the NIHR HPRUs in Healthcare Associated Infections and Antimicrobial Resistance, in partnership between the UKHSA and (i) Imperial College London [NIHR200876] and (ii) University of Oxford [NIHR200915].

The funder had no role in study design, data collection, data analysis, data interpretation, or writing of the paper.

\section{Transparency declarations}

None to declare. For the purpose of open access, the author has applied an 'Open Government Licence' to any Author Accepted Manuscript version arising.

\section{Disclaimer}

The views and opinions expressed in this paper are those of the authors and not necessarily those of the NIHR, the UKHSA or the Department of Health and Social Care.

\section{Supplementary data}

Figures S1 to S3 and Tables S1 to S3 are available as Supplementary data at JAC Online.

\section{References}

1 English surveillance programme for antimicrobial utilisation and resistance (ESPAUR) Report 2019-2020. https://assets.publishing.service.gov.uk/govern ment/uploads/system/uploads/attachment_data/file/936199/ESPAUR_Report_ 2019-20.pdf.

2 Dolk FCK, Pouwels KB, Smith DRM et al. Antibiotics in primary care in England: which antibiotics are prescribed and for which conditions? J Antimicrob Chemother 2018; 73: ii2-10.

3 Department of Health and Social Care. UK 5-year action plan for antimicrobial resistance 2019 to 2024. https://www.gov.uk/government/publications/ uk-5-year-action-plan-for-antimicrobial-resistance-2019-to-2024.

4 Bou-Antoun S, Costelloe C, Honeyford K et al. Age-related decline in antibiotic prescribing for uncomplicated respiratory tract infections in primary care in England following the introduction of a national financial incentive (the Quality Premium) for health commissioners to reduce use of antibiotics in the community: an interrupted time series analysis. J Antimicrob Chemother 2018; 73: 2883-92.

5 Smieszek T, Pouwels KB, Dolk FCK et al. Potential for reducing inappropriate antibiotic prescribing in English primary care. J Antimicrob Chemother 2018; 73: ii36-43.

6 Smith DRM, Dolk FCK, Pouwels KB et al. Defining the appropriateness and inappropriateness of antibiotic prescribing in primary care. J Antimicrob Chemother 2018; 73: ii11-ii18.

7 National Institute for Health and Care Excellence. Self-limiting respiratory tract and ear infections - antibiotic prescribing overview. https://path ways.nice.org.uk/pathways/self-limiting-respiratory-tract-and-ear-infectionsantibiotic-prescribing.

8 Dempsey PP, Businger AC, Whaley LE et al. Primary care clinicians' perceptions about antibiotic prescribing for acute bronchitis: a qualitative study. BMC Fam Pract 2014; 15: 194.

9 Horwood J, Cabral C, Hay AD et al. Primary care clinician antibiotic prescribing decisions in consultations for children with RTIs: a qualitative interview study. Br J Gen Pract 2016; 66: e207-13.

10 Langford BJ, So M, Raybardhan S et al. Antibiotic prescribing in patients with COVID-19: rapid review and meta-analysis. Clin Microbiol Infect 2021; 27: 520-31.

11 Langford BJ, So M, Raybardhan S et al. Bacterial co-infection and secondary infection in patients with COVID-19: a living rapid review and meta-analysis. Clin Microbiol Infect 2020; 26: 1622-9.

12 Gerver SM, Guy R, Wilson K et al. National surveillance of bacterial and fungal CO- and secondary infection in COVID-19 patients in England Lessons from the first wave. Clin Microbiol Infect 2021; 27: 1658-65.

13 Andrews A, Budd EL, Hendrick A et al. Surveillance of Antibacterial Usage during the COVID-19 Pandemic in England, 2020. Antibiotics 2021; 10: 841.

14 Rezel-Potts E, L'Esperance V, Gulliford MC. Antimicrobial stewardship in the UK during the COVID-19 pandemic: a population-based cohort study and interrupted time-series analysis. Br J Gen Pract 2021; 71: e331-8.

15 Public Health England. Coronavirus (COVID-19) in the UK: Testing in United Kingdom. https://coronavirus.data.gov.uk/details/testing.

16 Moriyama M, Hugentobler WJ, Iwasaki A. Seasonality of respiratory viral infections. Annu Rev Virol 2020; 7: 83-101.

17 Public Health England. Emergency Department: Syndromic Surveillance System: England Year 2021 Week 16. https://assets.publishing.service.gov.uk/ government/uploads/system/uploads/attachment_data/file/981848/EDSSS Bulletin2021wk16.pdf.

18 Public Health England. GP in Hours: Syndromic Surveillance System: England Year 2021 Week 16. https://assets.publishing.service.gov.uk/govern ment/uploads/system/uploads/attachment_data/file/981831/GPinHoursEng Bulletin2021Wk16.pdf.

19 Petersen I, Crozier A, Buchan I et al. Recalibrating SARS-CoV-2 antigen rapid lateral flow test relative sensitivity from validation studies to absolute sensitivity for indicating individuals shedding transmissible virus. Clin Epidemiol 2021; 13: 935-40.

20 Khalid TY, Duncan LJ, Thornton HV et al. Novel multi-virus rapid respiratory microbiological point-of-care testing in primary care: a mixed-methods feasibility evaluation. Fam Pract 2021; 38: 598-605. 